\title{
Many Triangulated Spheres
}

\author{
Gil Kalai* \\ Institute of Mathematics, Hebrew University, Jerusalem, Israel
}

\begin{abstract}
Let $s(d, n)$ be the number of triangulations with $n$ labeled vertices of $S^{d-1}$, the $(d-1)$-dimensional sphere. We extend a construction of Billera and Lee to obtain a large family of triangulated spheres. Our construction shows that $\log s(d, n) \geq C_{1}(d) n^{[(d-1) / 2]}$, while the known upper bound is $\log s(d, n) \leq C_{2}(d) n^{[d / 2]} \log n$.

Let $c(d, n)$ be the number of combinatorial types of simplicial $d$-polytopes with $n$ labeled vertices. (Clearly, $c(d, n) \leq s(d, n)$.) Goodman and Pollack have recently proved the upper bound: $\log c(d, n) \leq d(d+1) n \log n$. Combining this upper bound for $c(d, n)$ with our lower bounds for $s(d, n)$, we obtain, for every $d \geq 5$, that $\lim _{n \rightarrow \infty}(c(d, n) / s(d, n))=0$. The case $d=4$ is left open. (Steinitz's fundamental theorem asserts that $s(3, n)=c(3, n)$, for every n.) We also prove that, for every $b \geq 4, \lim _{d \rightarrow \infty}(c(d, d+b) / s(d, d+b))=0$. (Mani proved that $s(d, d+3)=$ $c(d, d+3)$, for every $d$.

Let $s(n)$ be the number of triangulated spheres with $n$ labeled vertices. We prove that $\log s(n)=2^{0.69424} n(1+o(1))$. The same asymptotic formula describes the number of triangulated manifolds with $n$ labeled vertices.
\end{abstract}

\section{Introduction}

How many triangulations with $n$ (labeled) vertices of $S^{d}$ (the $d$-dimensional sphere) are there? Let $s(d+1, n)$ denote the number of such triangulations.

\section{Theorem 1.1.}

$$
3 \log n\left(\begin{array}{c}
n-[(d+1) / 2] \\
{[d / 2]}
\end{array}\right) \geq \log s(d, n) \geq(1 /(n-d)(d+1))\left(\begin{array}{c}
n-[(d+2) / 2] \\
{[(d+1) / 2]}
\end{array}\right)
$$

\footnotetext{
* Research done, in part, while the author visited the mathematics research center at AT\&T Bell Laboratories.
} 
The upper bound for $s(d, n)$ follows from Stanley's upper bound theorem [41] for the numbers of faces of triangulated $d$-spheres with $n$ vertices. (Compare, Klee [26] and McMullen [34].)

Our main purpose is to construct a large family of triangulated spheres which demonstrates the lower bound.

We indicate two special cases of Theorem 1.1. For fixed $d \geq 3$,

$$
C_{1}(d) n^{[d / 2]} \log n \geq s(d, n) \geq C_{2}(d) n^{[(d-1) / 2]}
$$

Here, $C_{1}(d)$ and $C_{2}(d)$ are constants depending on $d$.

Define $b(z)=(1-z)^{1-z} / z^{2}(1-2 z)^{1-2 z}$. Let $x$ be a real number, $0<x<1$. Theorem 1.1 implies that $\log s([x n], n)=(b(x / 2))^{n(1+o(1))}$. Note that $b(x / 2)>0$ for every $0<x<1 . b(x / 2)$ is maximal and is equal to $1.61803 \ldots$ for $x=\left(1-5^{-1 / 2}\right)=$ $0.5528 \ldots$.

Let $s(n)$ be the total number of triangulated spheres (of all dimensions) on $n$ labeled vertices. The most accurate estimate we can give for $s(n)$ is $\left(C_{3}, C_{4}\right.$ are constants)

$$
C_{3} \log n n^{-1 / 2} 1.61803 \ldots{ }^{n} \geq \log s(n) \geq \log s(0.5528 \ldots n, n) \geq C_{4} n^{-2} 1.61803 \ldots{ }^{n} .
$$

Note that the number $\operatorname{simp}(n)$ of simplicial complexes on $n$ labeled vertices satisfies $\log \operatorname{sim} p(n)=2^{n(1+o(1))}$. More precisely, Kleitman [30] proved

$$
\log \operatorname{simp}(n)=\left(\begin{array}{c}
n \\
{[n / 2]}
\end{array}\right)(1+o(1))
$$

Recently, Korshunov [31] completely determined the asymptotic behavior of $\operatorname{simp}(n)$ !

The boundary complex of a simplicial $d$-polytope is a triangulated $(d-1)$ sphere. A triangulated sphere is polytopal if it is isomorphic to the boundary complex of a simplicial polytope.

Let $c(d, n)$ be the number of polytopal $(d-1)$-spheres with $n$ labeled vertices. Thus $c(d, n) \leq s(d, n)$. Denote $b=n-d$.

A fundamental theorem of Steinitz [44], [21, Chapter 13] asserts that every triangulated 2-sphere is polytopal. (Steinitz's theorem in its full generality asserts that every polyhedral 2 -sphere is isomorphic to the boundary complex of a 3-polytope.) Only a few decades after Steinitz's theorem was established, mathematicians have realized that nonpolytopal triangulated spheres do exist in higher dimensions. Simple examples of nonpolytopal triangulated 3-spheres with eight vertices were given by Grünbaum [20] and by Barnette [8]. The "Steinitz Problem"- the determination of polytopal spheres among all triangulated spheres-is one of the main problems in convex polytope theory. For a recent work on this subject, see Bokowski and Sturmfels [14].

Steinitz's theorem asserts that $s(3, n)=c(3, n)$. Works of Tutte [45], [46], Brown [15], and Richmond and Wormald [39] give good asymptotic estimates for $s(3, n)$ and exact formulas for related enumeration problems. 
An important result of Mani [33] asserts that every triangulated $(d-1)$-sphere with at most $(d+3)$-vertices is polytopal. (Kleinschmidt [28] extended Mani's result and proved that every polyhedral $(d-1)$-sphere with at most $d+3$ vertices is polytopal.) There are exactly $[d / 2]$ simplicial $d$-polytopes with $d+2$ vertices and the value of $c(d, d+3)(=s(d, d+3))$ was determined by Perles. (See Chapter 6 of [21].)

In the last 20 years extensive work has been done on the enumeration of triangulated 3-spheres and simplicial 4-polytopes with few vertices. It was proved that $c(4,8)=37$ (Grünbaum and Sreedharan [22], correcting Brückner [16]), $s(4,8)=39$ [9], $c(4,9)=1142$, and $s(4,9)=1296$ [3], [6]. For further results of this type, see [2], [4], and [5].

It was conjectured by several people that for every fixed $d \geq 4$, "most" triangulated $(d-1)$-spheres are not polytopal, i.e., that $\lim _{n \rightarrow \infty}(c(d, n) / s(d, n))=0$. Similarly, it was believed that for every $b, b \geq 4, \lim _{d \rightarrow \infty}(c(d, d+b) / s(d, d+b))=$ 0 .

Goodman and Pollack [18], [19] have recently proved that $\log c(d, n) \leq$ $d(d+1) n \log n$. This remarkable result is proved using a theorem of Milnor which gives bounds on the sum of the Betti numbers of real algebraic varieties. Alon [1] extended their result to arbitrary polytopes.

Goodman and Pollack's upper bound on $c(d, n)$ combined with our lower bound on $s(d, n)$ imply (in a very strong sense) that for every $d \geq 5$, $\lim _{n \rightarrow \infty}(c(d, n) / s(d, n))=0$. A proof that most triangulations of the 3 -sphere are not polytopal is still unknown.

Our construction gives the bound $\log s(d, d+b)>D(b) \cdot d^{b-2}$, while the method of proof of Goodman and Pollack's theorem gives $\log c(d, d+b) \leq$ $b(b-1) d \log d$ (see [1]). This shows that for every $b \geq 4$,

$$
\lim _{d \rightarrow \infty}(c(d, d+b) / s(d, d+b))=0 .
$$

By Goodman and Pollack's bound the total number of polytopal triangulated spheres with $n$ labeled vertices is bounded by $2^{(n+1)^{3}}$. (Alon's result gives the same bound for the nonsimplicial case.) Thus, the gap between the numbers of triangulated spheres and polytopal spheres is most striking when $d=[x n]$ for some constant $x$.

For example, the number of triangulated spheres with 1000 labeled vertices is more than $2^{2^{650}}$, but less than $2^{2^{30}}$ out of them are polytopal. The number of triangulated spheres with 1000000 vertices is between $2^{2^{694,200}}$ and $2^{2^{694,250}}$, but less than $2^{2^{62}}$ out of them are polytopal.

Our construction is a modification of a construction of Billera and Lee [10], [11]. For a simplicial complex $C, f_{k}(C)$ denotes the number of $k$-dimensional faces of $C$. The vector $f(C)=\left(f_{0}(C), f_{1}(C), \ldots\right)$ is called the f-vector of $C$. The same definition applies for polytopes. McMullen [35] proposed a complete characterization of $f$-vectors of simplicial $d$-polytopes. Billera and Lee built, for every vector $f$ of nonnegative integers which satisfies McMullen's conditions, a shellable $(d-1)$-sphere with $f$ as its $f$-vector. They further realized this sphere as a boundary complex of a simplicial $d$-polytope, thus proving the sufficiency part of McMullen's conjecture. Stanley [43] proved the necessity part of McMullen's conjecture using deep results from algebraic-geometry. 
Billera and Lee's $(d-1)$-spheres as well as our more general class of spheres are obtained as the boundaries of shellable balls spanned by some of the facets $(=d$-faces) of a cyclic $(d+1)$-polytope. Specifically, let $C(d+1, n)$ denote the cyclic $(d+1)$-polytope with $n$ vertices. Billera and Lee considered the (reverse) lexicographic order on the facets of $C(d+1, n)$, and studied simplicial complexes spanned by initial sets of facets. They proved that simplicial complexes obtained in this way are actually shellable $d$-balls, and their boundaries are polytopal $(d-1)$-spheres. We consider a certain partial order of the facets of $C(d+1, n)$, and study simplicial complexes which are spanned by initial sets of facets. All these complexes are shellable $d$-balls and their boundaries form a large class of triangulated $(d-1)$-spheres, which we call squeezed spheres. Squeezed spheres demonstrate the lower bounds in (1.1).

McMullen's conditions are conjectured to hold for arbitrary triangulated spheres. They hold almost trivially for squeezed spheres. Results by Lee [32] on the diameter of the Billera-Lee polytopes (related to the "Hirsch conjecture") also extend to arbitrary squeezed spheres.

We hope that squeezed spheres will play a role among triangulated spheres similar to that which shifted complexes play among simplicial complexes. (See [24] and [13].) In particular, a squeezing operation for spheres is now desirable.

Problems which remains open are to construct many triangulations (say, $2^{\mathrm{Cn}^{2}}$ ) for the three-dimensional sphere, and to give an explicit example of a nonpolytopal (say, four-dimensional) squeezed sphere.

\section{Preliminaries}

\subsection{Orderings}

For an integer $n,[n]$ denotes the set $\{1,2, \ldots, n\} . \mathbb{N}$ will denote the set of positive integers. For a set $A$ and a nonnegative integer $d, A^{(d)}$ is the set of $d$-subsets of $A$. The partial order $<_{p}$ on $\mathbb{N}^{(d)}$ is defined as follows: if $S, T \in \mathbb{N}^{(d)}, S=\left\{i_{1}, \ldots, i_{d}\right\}$, $i_{1}<\cdots<i_{d}$, and $T=\left\{j_{1}, \ldots, j_{d}\right\}, j_{1}<\cdots<j_{d}$, then $S \leq_{\mathrm{p}} T$ iff for every $1 \leq k \leq$ $d, i_{k} \leq j_{k}$.

Define the lexicographic order $<_{\mathrm{L}}$ on $\mathbb{N}^{(d)}$ as follows: for $S, T \in \mathbb{N}^{(d)}, S<_{\mathrm{L}} T$ if $\min (S \Delta T) \in S$. The reverse lexicographic order $<_{\mathrm{RL}}$ is defined by $S<_{\mathrm{RL}} T$ if $\max (S \Delta T) \in T$.

\subsection{Simplicial Complexes}

We follow the definitions and notation of Billera and Lee [11]. We repeat here some basic definitions.

Let $C$ be a simplicial complex, and let $\nu$ be a vertex not in $C$. The cone over $C$ with apex $\nu$ is defined by

$$
\operatorname{cone}(C, \nu)=C \cup\{S \cup\{\nu\}: S \in C\}
$$


A simplicial complex is pure if all its maximal faces have the same size. Maximal faces of a pure simplicial complex are called facets. Two facets $S$ and $T$ of a pure simplicial complex are adjacent if they intersect in a maximal proper face of each.

Let $C$ be a pure simplicial complex and let $S$ and $T$ be two facets of $C$. The distance between $S$ and $T, d(S, T)$, is the minimal integer $k$ such that there exists a sequence of facets $S=F_{0}, F_{1}, \ldots, F_{k}=T$ with the property that $F_{i}$ is adjacent to $F_{\mathrm{i}+1}$ for every $1 \leq \mathrm{i}<k$. (If no such sequence exists $d(S, T)=\infty$.) The diameter of $C, \Delta(C)$, is the maximal value of $d(S, T)$ taken over all pairs of facets of $C$. A pure simplicial complex $C$ is strongly connected if it has a finite diameter.

A $d$-pseudomanifold $C$ is a strongly connected $d$-dimensional simplicial complex, such that every $(d-1)$-face is included in at most two facets. The boundary of $C, \partial C$, is the $(d-1)$-dimensional simplicial complex spanned by those $(d-1)$-faces of $C$ which are included in exactly one facet of $C$.

\subsection{Shelling}

A pure $(d-1)$-dimensional simplicial complex $C$ is shellable if its maximal faces can be ordered $F_{1}, F_{2}, \ldots, F_{t}$, so that for every $i$ and $k, 1 \leq i<k \leq t$, there exists $j, 1 \leq j \leq k$, such that $F_{1} \cap F_{k} \subset F_{j} \cap F_{k}$ and $F_{j}$ is adjacent to $F_{k}$. Such an ordering is called a shelling order of $C$.

\subsection{Vertex Decomposability and the Hirsch Condition (needed for Section 5)}

Let $C$ be a pure $d$-dimensional simplicial complex. $C$ satisfies the Hirsch condition if $\Delta(C) \leq f_{0}(C)-d$. (Recall that $f_{0}(C)$ is the number of vertices of $C$.) The Hirsch conjecture asserts that every polytopal sphere satisfies the Hirsch condition. (See Klee and Kleinschmidt [27] for a recent survey on this conjecture.)

Provan and Billera [38] related the Hirsch condition to a strong form of shellability called vertex-decomposability.

Vertex-decomposable simplicial complexes are defined recursively as follows: $C=\{\varnothing\}$ is vertex-decomposable. A pure simplicial complex $C$ is vertex-decomposable if there exists a vertex $\nu \in C$ (called a shedding vertex) such that $l k(v, C$ ) and $\operatorname{ast}(v, C)$ are vertex-decomposable. (Here, $l k(v, C)=\{S \backslash\{v\}: S \in C, v \in S\}$ and $\operatorname{ast}(v, S)=\{S \in C: v \notin S\}$.)

A pure simplicial $(d-1)$-dimensional complex $C$ is weakly vertex-decomposable if $C$ is a single $(d-1)$-simplex together with its faces, or else there is a vertex $v$ of $C$ (a weak shedding vertex) such that $\operatorname{ast}(v, C$ ) is a weakly vertexdecomposable $(d-1)$-dimensional complex.

Theorem 2.1 (Provan and Billera [38]).

(i) Every vertex-decomposable simplicial complex satisfies the Hirsch condition.

(ii) The diameter of a weakly vertex-decomposable $(d-1)$-dimensional simplicial complex with $n$ vertices is at most $2(n-d)$, twice the Hirsch bound. 


\section{Squeezed Balls and Spheres}

Let $d>0$ be a fixed odd integer. Define a collection $F_{d}$ of $(d+1)$-subsets of $\mathbb{N}$ as follows: put $e=(d+1) / 2$. For $F \in \mathbb{N}^{(d+1)}, F \in F_{d}$ iff $F=\left\{i_{1}, i_{1}+1\right\} \cup$ $\left\{i_{2}, i_{2}+1\right\} \cup \cdots \cup\left\{i_{e}, i_{e}+1\right\}$, where $i_{1} \geq 1$ and for every $j, e>j \geq 1, i_{j+1}>i_{j}+1$. Define $F_{d}(n)=F_{d} \cap[n]^{(d+1)}$. Let $I$ be an initial set of $F_{d}$ with respect to the partial order $<_{p}$ on $\mathbb{N}^{(d+1)}$ and let $B(I)$ be the simplicial complex spanned by $I$. We will prove that $B(I)$ is a shellable $(d+1)$-ball.

For $d$ even put $F_{d}=\left\{\{0\} \cup F: F \in F_{d-1}\right\}$. For an initial set $I$ of $F_{d}$ with respect to the partial ordering, let $B(I)$ be the simplicial complex spanned by $I$. We will show that in this case $B(I)$ is also a shellable ball. In fact, for $d$ even and $I$ an initial set of $F_{d}, B(I)$ is easily seen to be a cone over some $B(J)$, where $J$ is an initial set of $F_{d-1}$. We call a simplicial complex $B(I)$ of the form described above a squeezed ball. The boundary of a squeezed ball is called a squeezed sphere.

Remark. $\quad F_{d}(n)$ can be regarded as a subcollection of the facets of the cylic $(d+1)$-polytope with $n$ vertices.

Theorem 3.1. Let $d \geq 1$ be a fixed integer. Let 1 be an initial set of $F_{d}$ with respect to the partial order $<_{\mathrm{p}}$. Let $B(I)$ be the simplicial complex spanned by $I$. Then any ordering $F_{1}, F_{2}, \ldots, F_{1}$ of I which extends the partial order $<_{p}$, is a shelling order for $B(I)$.

Proof. The proof is similar to the proof of Lemma 4 in Billera and Lee [11]. We will consider the case of odd $d$. (The proof for even $d$ is the same.) Let $F_{1}$, $F_{2}, \ldots, F_{1}$ be an ordering of $I$ which extends the partial order $<_{p}$. Let $F_{k}=$ $[1, d+1-2 p] \cup\left\{i_{1}, i_{1}+1\right\} \cup \cdots \cup\left\{i_{p}, i_{p}+1\right\}$ where $i_{1}>d+2-2 p$ and $i_{j+1}>i_{j}+1$ for $p>j \geq 1$. Define, for $1 \leq j \leq p, G_{k}^{j}=F_{k} \backslash\left\{i_{j}+1\right\} . G_{k}^{j}$ is a $(d-1)$-face of $F_{k}$. If $u_{j}$ is the maximal element in $\left[i_{j}\right] \backslash F_{k}$ then $G_{k}^{j} \cup\left\{u_{j}\right\}$ is a $d$-face in $F_{d}$ which precedes $F_{k}$ in the partial order.

It remains to show that if $S=F_{k} \cap F_{i}$ for $i<k$ then $S \subset G_{k}^{j}$ for some $j, p \geq j \geq 1$. If $S \not \subset G_{k}^{\prime}$ for every $j, p \geq j \geq 1$ then $S \supset R=\left\{i_{1}+1, \ldots, i_{p}+1\right\}$. But it is easy to see that every $F \in F_{d}$ which includes $R$ satisfies $F \geq_{\mathrm{p}} F_{k}$. Thus, $F_{i} \geq_{\mathrm{p}} F_{k}$. A contradiction.

Corollary 3.2. $B(I)$ is a shellable ball.

Proof. It is easy to see that every $(d-1)$-face of $B(I)$ is included in at most two $d$-faces. Thus, $B(I)$ is a shellable pseudomanifold and as is well known (see Danaraj and Klee [17]). $B(I)$ is either a shellable sphere or a shellable ball. It is easy to see that there is a $(d-1)$-face of $B(I)$ which is included in exactly one $d$-face. Hence $B(I)$ is a shellable ball.

Since squeezed spheres are boundaries of shellable balls, they are PL-spheres. We do not know if they are, in general, shellable. (A recent result of Pachner [37] asserts that every PL-sphere is the boundary of some shellable ball.) 
The construction of Billera and Lee is the special case where $I$ is an initial set of $F_{d}$, with respect to the reverse lexicographic order. (They also consider only a subset of $F_{d}$ for odd $d$.) We will refer to the triangulated spheres considered by Billera and Lee as compressed spheres. Billera and Lee proved that compressed spheres are polytopal. We refer to the corresponding polytopes as Billera-Lee polytopes.

Proposition 3.3. Let $B_{1}, B_{2}$ be two squeezed d-balls. If $\partial B_{1}=\partial B_{2}$ then $B_{1}=B_{2}$.

Proof. Let $B$ be a squeezed $d$-ball and let $S$ be a facet of $\partial B$. $S$ is included in exactly one facet of $B$. Note that $S$ is included in at most two members of $F_{d}$. Note also that if $S$ is included in two members of $F_{d}$, say $F_{1}$ and $F_{2}$, then they are comparable with respect to the partial order $<_{p}$. Define $F(S)$ to be the minimal set in $F_{d}$ with respect to $<_{p}$ which contains $S$. From the discussion above it follows that $F(S)$ is defined and belongs to $B$. Define now $\hat{B}=$ $\{F(S):|S|=d-1, S \in \partial B\} \subset B$. Let $F$ be a facet of $B$ which is maximal in $B$ with respect to $<_{p}$ and let $u=\min F$. Then $S=(F \backslash\{u\}) \in \partial B$ and $F=F(S) \in \hat{B}$. Therefore, $B=\left\{F \in F_{d}: F \leq_{\mathrm{p}} \hat{F}, \hat{F} \in \hat{B}\right\}$.

Proposition 3.4. Let $S(I)=\partial B(I)$ be a squeezed sphere. Then if $i$ is a vertex of $S(I)$ and $j \leq i$ then $j$ is a vertex of $S(I)$.

Proof. Since $I$ contains a set which contains $i, I$ also contains a set which contains $j$. Consider $F \in I$, which is maximal (with respect to the partial order) among sets in $I$ which contain $j$. It is easy to see that for some $k \in S, k \neq j, F \backslash\{k\} \in$ $S(I)$.

Remark. Let $P$ be a ranked poset. A subset $Q$ of $P$ is a ranked subposet of $P$ if $Q$ forms a ranked poset whose rank function is the restriction from $P$ to $Q$ of the rank function of $P$. Clearly every initial set in $P$ is a ranked subposet.

Let $I$ be a ranked subposet of $F_{d}$ and let $B(I)$ be the simplicial complex spanned by $I$. The proof of Theorem 3.1 can be directly extended to show that $B(I)$ is a shellable ball. This construction does not improve substantially the lower bounds for $s(d, n)$.

\section{The Number of Triangulated Balls and Spheres}

In this section we will estimate the number $s(d, n)$ of triangulated $(d-1)$ spheres with $n$ vertices. We will also consider the number $m(d, n)$ of triangulated $(d-1)$-manifolds with $n$ vertices.

We need the following lemma. The proof is an easy application of Stirling's estimation of $n$ ! 


\section{Lemma 4.1.}

(i) Define $b(x)=(1-x)^{1-x} / x^{x}(1-2 x)^{1-2 x}$. Then for a fixed real number $x, \quad 0<x<\frac{1}{2}, \quad\left(\begin{array}{l}n-[x n] \\ {[x n]}\end{array}\right)=c(x) n^{-(1 / 2)}(b(x))^{n}(1+o(1)) . \quad$ (Here, $\quad c(x)=$ $(1-x)^{1 / 2}(2 \pi x(1-2 x))^{-1 / 2}$.

(ii) $b(x)$ attains its maximum at $x_{\max }=\left(1-5^{-1 / 2}\right) / 2$.

\subsection{The Lower Bounds}

We now give the lower bounds obtained by the family of squeezed spheres.

Let $f(d, n)$ be the number of initial sets in $F_{d}(n)$. It is clear that for even $d$, $f(d, n)=f(d-1, n-1)$. We will therefore assume that $d$ is odd. As before let $e=(d+1) / 2$. Let $s q(d, n)$ be the number of squeezed $(d-1)$-spheres with $n$ vertices.

$F_{d}$ is a ranked poset, for $F=\left\{i_{1}, i_{1}+1\right\} \cup \cdots \cup\left\{i_{e}, i_{e+1}\right\} \in F_{d} \cdot r(F)=\sum_{j=1}^{e} i_{j}-e^{2}$. Thus, for $F \in F_{d}(n), 0 \leq r(F) \leq(n-2 e) e$.

Let $a(n, k, r)$ be the number of ways to represent $r$ as a sum $r=a_{1}+a_{2}+\cdots+a_{k}$ where $0 \leq a_{1} \leq a_{2} \leq \cdots \leq a_{k} \leq n$. Define $a(n, k)=\max \{a(n, k, r): r \geq 0\}$. Clearly, $a(n, k, r)=0$ for $r>k n$. Also $\sum_{r \geq 0} a(n, k, r)=\left(\begin{array}{c}n+k \\ k\end{array}\right)$. Therefore $a(n, k) \geq$ $1 /(1+k n)\left(\begin{array}{c}n+k+1 \\ k\end{array}\right)$.

Remark. It is well known (see, for example, Chapter 3 of Andrews [7] that $a(n, k, r)$ is the coefficient of $q^{r}$ in $\left[\begin{array}{c}n+k \\ k\end{array}\right](q)$. It is also known that the sequence $a(n, k, 0), a(n, k, 1), \ldots, a(n, k, k n)$ is symmetric and unimodal, hence, $a(n, k)=$ $a(n, k,[k n / 2])$.

Let $b(n, d, r)=\left|\left\{S \in F_{d}(n): r(S)=r\right\}\right|$ and $b(n, d)=\max \{b(n, d, r): r \geq 0\}$. A simple inspection shows that $b(n, d, r)=a(n-1-d, e, r)$. Thus, $b(n, d)=$ $a(n-1-d, e)$.

Theorem 4.2. Put $e=[(d+1) / 2]$ and $e^{\prime}=[(d+2) / 2]$.

$$
\log s(d, n) \geq \log s q(d, n) \geq(1 /(n-d) e)\left(\begin{array}{c}
n-e^{\prime} \\
e
\end{array}\right)-1 \text {. }
$$

In particular:

(i) Let $d \geq 2$ be a fixed integer. Then $\log s q(d, n) \geq C_{2}(d) n^{[(d+1) / 2]-1}$.

(ii) Let $b \geq 2$ be a fixed integer. Then $\log s q(d, d+b) \geq C_{4}(b) d^{b-2}$.

(iii) $\log s q([x n], n) \geq C_{6}(x) n^{-(5 / 2)}(b(x / 2))^{n}$.

Proof. To prove (4.1) it is enough to consider the case that $d$ is odd. The number of squeezed $d$-balls with at most $n$ vertices is exactly $f(d, n)$. By Proposition 3.3, this is also the number of squeezed $(d-1)$-spheres with at most $n$ vertices. By Proposition 3.4, the number $s q(d, n)$ of squeezed $(d-1)$-spheres with exactly $n$ 
vertices is equal to the number of squeezed $d$-balls with exactly $n$ vertices, namely $f(d, n)-f(d, n-1)$. Now, $f(d, n) \geq 2^{b(n, d)}=2^{a\left(n-1-d_{,} e\right)}$. Since, for $n>k>1$, $a(n, k)>a(n-1, k)$ we obtain, for odd $d \geq 3$,

$$
\log s(d, n) \geq \log s q(d, n)>a(n-1-d, e)-1 \geq(1 /(n-d) e)\left(\begin{array}{c}
n-e \\
e
\end{array}\right)-1
$$

Remark. With some more effort it can be shown that

$$
\log s q([x n], n) \geq a(n-1-d, e)-1 \geq C_{8}(x) n^{-2}(b(x / 2))^{n}(1+o(1)) .
$$

\subsection{The Upper Bounds}

Here are the upper bounds on $s(d, n)$ and $m(d, n)$ which follow from the upper bound for the number of facets (= maximal faces) of triangulated spheres. The upper bound theorem for triangulated spheres (proved by Stanley [41]) asserts that a triangulated $(d-1)$-sphere $C$ with $n$ vertices has at most $\left(\begin{array}{c}n-[(d+1) / 2)] \\ n-d\end{array}\right)+\left(\begin{array}{c}n-[(d+2) / 2] \\ n-d\end{array}\right)(d-1)$-faces. This expression is smaller than $2\left(\begin{array}{c}n-[(d+1) / 2] \\ {[d / 2]}\end{array}\right)$.

The number of pure $(d-1)$-dimensional simplicial complexes with $n$ labeled

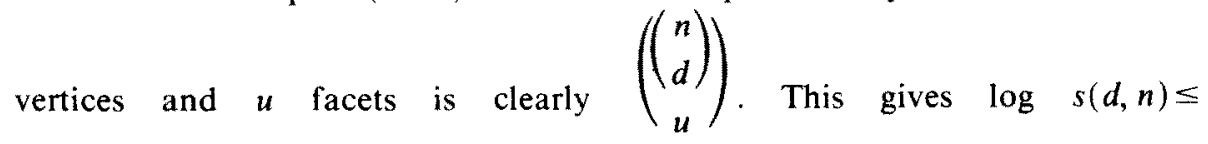
$2 d \log n\left(\begin{array}{c}n-[(d+1) / 2] \\ {[d / 2]}\end{array}\right)+1$. We can gain a factor of $d$ in this expression by using the following:

Proposition 4.3. Let $p m(d, n, u)$ be the number of $(d-1)$-pseudomanifolds with $n$ vertices and $u$ facets. Then $p m(d, n, u) \leq n^{u+d}$.

Proof. Put $t=d+u-1$. Let $C$ be a $(d-1)$-pseudomanifold on the vertex set [n]. Associate to $C$ an integral vector $x(C)=\left(x_{1}, \ldots, x_{1}\right)$ which satisfies $1 \leq x_{i} \leq n$ for every $1 \leq i \leq t$ and a chain of subcomplexes $C_{1}, \ldots, C_{u}$ as follows: $x_{1}, \ldots, x_{d}$ are the vertices of the lexicographically first facet $F$ of $C$, and $C_{1}$ is the complex spanned by $F$. Assume that $x_{i}, i \leq k$, and $C_{1}, j \leq k-d+1$, are defined. Let $R$ be the lexicographically minimal $(d-2)$-face in the boundary of $C_{k-d+1}$ and let $S$ be the facet of $C$, not in $C_{k-d+1}$ which contains $R$. Define $x_{k+1}$ by $\left\{x_{k+1}\right\}=S \backslash R$ and define $C_{k-d+2}$ to be the complex spanned by $C_{k-d+1} \cup\{S\}$. As easily seen, $x(C)$ determines $C$.

Stanley's theorem implies that a triangulated $(d-1)$-manifold with $n$ vertices has at most $2 n d^{-1}\left(\begin{array}{c}n-[d / 2]-1 \\ {[(d-1) / 2]-1}\end{array}\right)$ facets. 
We summarize the asymptotic upper bounds for $s(d, n)$ and $m(d, n)$ which are obtained from the upper bound theorems by Proposition 4.3:

\section{Theorem 4.4.}

$$
\begin{gathered}
\log s(d, n) \leq 2 \log n\left(\begin{array}{c}
n-[(d+1) / 2] \\
{[d / 2]}
\end{array}\right)+d \\
\log m(d, n) \leq 2 n d^{-1} \log n\left(\begin{array}{c}
n-[d / 2]-1 \\
{[(d-1) / 2]}
\end{array}\right)+d
\end{gathered}
$$

In particular;

(i) Let $d \geq 2$ be a fixed integer. Then $\log s(d, n) \leq C_{1}(d) \log n n^{[d / 2]}$ and $\log m(d, n) \leq C_{3}(d) \log n n^{[(d+1) / 2]}$.

(ii) Let $b \geq 2$ be fixed. Then $\log s(d, d+b) \leq \log m(d, d+b) \leq$ $C_{5}(b) \log d d^{b+1}$.

(iii) Let $x$ be a fixed real number $0<x<1$. Then, $\log s([x n], n) \leq$ $\log m([x n], n) \leq C_{7}(x) n^{(-1 / 2)}(b(x / 2))^{n}(1+o(1))$.

Theorem 1.1 is a combination (in a weaker but more elegant form) of (4.1) and (4.3).

\section{Further Properties of Squeezed Balls and Spheres}

\subsection{Squeezed Spheres and the "Hirsch Conjecture"}

Proposition 5.1 (Compare Lee [32]).

(i) Squeezed balls satisfy the Hirsch condition.

(ii) Let $d$ be even. For a squeezed $(d-1)$-sphere $S, \Delta(S) \leq f_{0}(S)-d+1$.

(iii) Let $d$ be odd. For a squeezed $(d-1)$-sphere $S, \Delta(S) \leq 2\left(f_{0}(S)-d\right)$.

(iv) Squeezed 3-spheres satisfy the Hirsch condition.

Proof. The proofs of Lee [32] of the special case of compressed spheres extend directly. By the Provan-Billera's theorem (Theorem 2.1(i)) in order to prove part (i) it is enough to show that squeezed balls are vertex-decomposable. Indeed, if $B$ is a squeezed $d$-ball then $l k(1, B)$ and $a s t(1, B)$ are combinatorially isomorphic to squeezed balls (of dimensions $d-1$ and $d$, respectively).

For the proof of (ii) see the argument in Lee [32]. (The argument applies to boundaries of those squeezed balls which are cones.)

To prove (iii) it is left by Theorem 2.1 (ii) to show that squeezed spheres are weakly vertex-decomposable. The proof is identical to the following unpublished proof by Lee for compressed spheres, which preceded his stronger published result. Let $l$ be an initial set of $F_{d}$ and let $n$ be the maximal vertex of $I$. We prove by induction on $n$. The case $n=d+1$ is easy, so assume $n>d+1$. Let $\hat{I}$ be an initial set in $F_{d}$ consisting of those $F$ in $I$ that do not contain $n$. Compare 
the facets of $\partial B(I)$ and $\partial \mathrm{B}(\hat{I})$. It is easy to see from the construction that each facet of $\partial B(\hat{I})$ that is not in $\partial B(I)$ contains $n-1$, and that each facet of $\partial B(I)$ that is not in $\partial B(\hat{I})$ contains $n$. Hence the facets of ast $(n-1, \operatorname{ast}(n, \partial B(I)))$ are precisely those of ast $(n-1, \partial B(\hat{I}))$, the latter being weakly vertex-decomposable by induction. So ast $(n, \partial B(I))$ is weakly vertex-decomposable with a weak shedding vertex $n$.

Squeezed 3-spheres are obtained from squeezed 3-balls by forming a cone over the boundary. Part (iv) is therefore a consequence of (i) and the fact that every triangulated 2-sphere satisfies the Hirsch condition.

\subsection{Squeezed Spheres and the "g-Conjecture"}

Our next results are of a technical nature. We point out that $f$-vectors of squeezed spheres satisfy McMullen's conditions (which are conjectured to hold for all triangulated spheres.) For the definitions of the $h$-vector, an $M$-vector, and the assertion of the " $g$-conjecture" we refer the reader to Billera and Lee [11] or Stanely [43].

Let $d$ be fixed. Let $e=[(d+1) / 2]$, and let $\Phi^{(e)}$ be the set of all monomials of degree at most $e$ in the variables $Y_{1}, Y_{2}, \ldots$ Put $Y_{0}=1$.

A bijection $\alpha: F_{d} \rightarrow \Phi^{(e)}$ is defined as in Billera and Lee [11]: for $F \in F(n)$, $F=V_{d} \cup\left\{i_{1}, i_{1}+1\right\} \cup \cdots \cup\left\{i_{e}, i_{e}+1\right\}$. (Here, $V_{d}=\{\varnothing\}$ for odd $d$ and $V_{d}=\{0\}$ for $d$ even.) $\alpha(F)=Y_{u_{1}} \cdot Y_{u_{2}} \cdots Y_{u_{e}}$ where $u_{j}=i_{j}-2 j+1$. Note that if $F=V_{d} \cup\{1,2 e-2 p\} \cup\left\{j_{1}, j_{1}+1\right\} \cup \cdots \cup\left\{j_{p}, j_{p}+1\right\}$ where $j_{1}>2 e-2 p+1$ then $\operatorname{deg} \alpha(F)=p$.

Proposition 5.2. The f-vector of squeezed spheres satisfies McMullen's conditions.

Proof. Let $I$ be an initial set in $F$. Let $M(I)=\alpha(I) \subset \Phi^{(n)} . M(I)$ is an order ideal of monomials. Let $k_{i}=|\{m \in M(I): \operatorname{deg} m=i\}| . k_{1}, k_{2}, \ldots$, is an $M$-vector. The shelling of $B(I)$ demonstrates that $\left.h_{i}(B)(I)\right)=k_{i}$ for $o \leq i \leq e$ and $h_{i}(B(I))=$ 0 for $i>e$. The $h$-vector of $S(I)=\partial B(I)$ is determined by the $h$-vector of $B(I)$ by $h_{i}(S(I))-h_{i-1}(S(I))=h_{i}(B(I))-h_{d+1-i}(B(I))=h_{i}(B(I))$. (See [11].) This proves that $\left(1, h_{1}(S(1))-h_{0}(S(I)), h_{2}(S(I))-h_{1}(S(I)), \ldots, h_{e-1}(S(I))-\right.$ $\left.h_{e}(S(I))\right)$ is an $M$-vector.

A triangulated $(d-1)$-sphere is $k$-stacked if it is the boundary of a triangulated $d$-ball with the same $(d-k-1)$-skeleton.

Proposition 5.3. Let $S$ be a squeezed $(d-1)$-sphere, then (i) $S$ is e-stacked, and (ii) if $h_{k}(S)=h_{k+1}(S)$ for some $0 \leq k \leq[d / 2]-1$, then $S$ is $k$-stacked.

The proofs are the same as the proofs for the compressed case by Billera and Lee [11] (for (i)) and by Kleinschmidt and Lee [29] (for (ii)).

Remarks. 1. Stanley [42] proved that every e-stacked $(d-1)$-sphere satisfies McMullen's conditions. 
2. A $d$-polytope $P$ is $k$-stacked if it can be triangulated without introducing new $j$-faces for $j \leq d-k-1$.McMullen and Walkup [36] conjectured that every simplicial $d$-polytope $P$ that satisfies $h_{k}(P)=h_{k+1}(P)$ for some $k, i \leq k \leq[d / 2]-1$, is a $k$-stacked polytope. Kleinschmidt and Lee [29] proved the assertion of this conjecture for the Billera-Lee polytopes.

\section{Open Problems}

\subsection{Properties of Squeezed Spheres}

1. Which squeezed $d$-spheres are polytopal? For $d \geq 4$ very few of them are. Yet, I do not know, at present, any explicit example of a nonpolytopal squeezed sphere.

Perhaps all squeezed 3-spheres are polytopal (in analogy with Proposition 9.2 of [23].)

2. Are squeezed spheres shellable?

\subsection{More Precision}

3. For fixed $d$ and $n \rightarrow \infty$ there is still a substantial gap between the lower and upper bounds of $\log s(d, n)$, especially for $d$ even. I believe that the value of $s(d, n)$ is closer to the upper bounds in (1.2). It can be shown that our lower bounds cannot be substantially improved by considering balls spanned by subsets of facets of cyclic polytopes.

We left a large gap between the lower and upper bounds for $m(d, n)$. I believe that in this case also the truth is closer to the upper bound.

Most urgently, we would like to estimate the number of traingulated 3-spheres with $n$ vertices and the number of triangulated 2 -manifolds with $n$ vertices. I expect that $2^{c n^{2}}$ is a lower bound for both these questions.

4. How many topologically distinct triangulated $d$-manifolds on $n$ vertices are there? For $d=2$ the answer is $C n^{2}$. For $d=3$ the answer is somewhere between $n^{2}$ and $2^{n^{2}}$.

\subsection{Greater Generality}

5. Let $f=\left(f_{0}, \ldots, f_{d-1}\right)$ be a vector of nonnegative integers. Let $s(f)$ be the number of triangulated $(d-1)$-spheres with $f$ as their $f$-vector. Estimate $s(f)$.

Note that for $d$ even there is only one neighborly squeezed $(d-1)$-sphere, namely, that corresponding to the cyclic $d$-polytope. We expect, however, that the number $s n(d, n)$ of neighborly $(d-1)$-spheres with $n$ vertices is very large. Perhaps for fixed even $d, \lim _{n \rightarrow \infty}(\log s n(d, n) / \log s(d, n))=1$. A large number of neighborly polytopes was constructed by Shemer [40].

In some cases, estimating $s(f)$ is related to structural properties of triangulated spheres with $f$ as their $f$-vector [25]. 
6. It is possible that the upper bounds for $s(n)$ and $s(d, n)$ apply to arbitrary ranked Eulerian posets (of rank $d+1$ and $n$ atoms). This requires a far-reaching extension of the upper bound theorem which is still unknown. By Klee's upper bound theorem [26], the upper bound in (1.2) applies to arbitrary Eulerian $(d-1)$-dimensional simplicial complexes.

\section{Acknowledgments}

I am thankful to N. Alon, L. J. Billera, C. W. Lee, N. Linial, and M. A. Perles for helpful comments.

\section{References}

1. N. Alon, The number of polytopes, configurations and real matroids, Mathematika 33 (1986), $62-71$.

2. A. Altshuler, Neighborly 4-polytopes and neighborly combinatorial manifolds with ten vertices, Canad. J. Math. 29 (1977), 400-420.

3. A. Altshuler and 1 . Steinberg, An enumeration of combinatorial 3-manifolds with nine-vertices, Discrete Math. 16 (1976), 113-137.

4. A. Altshuler and L. Steinberg, Enumeration of the quasi-simplicial 3-spheres and 4-polytopes with eight vertices, Pacific J. Math. 113 (1984), 269-288.

5. A. Altshuler and L. Steinberg, The complete enumeration of the 4-polytopes and 3-spheres with eight vertices, Pacific $J$. Math. 118 (1985), 1-16.

6. A. Altshuler, J. Bokowski, and L. Steinberg. The classification of simplicial 3 -spheres with nine vertices into polytopes and non-polytopes, Discrete Math. 31 (1980), 115-125.

7. G. Andrews, The Theory of Partitions, Addison-Wesley, Reading, MA, 1976.

8. D. Barnette, Diagrams and Schlegel diagrams, in Combinatorial Structures and Their Applications (R. Guy, H. Hanani, N. Sauer, and J. Schonheim, eds.), 1-5, Gordon and Breach, New York, 1970.

9. D. Barnette, The triangulations of the 3 -sphere with up to 8 vertices, J. Combin. Theory Ser. A 14 (1973), 37-52.

10. L. Billera and C. Lee, Sufficiency of McMullen's conditions for $f$-vectors of simplicial polytopes, Bull. Amer. Math. Soc. 2 (1980), 181-185.

11. L. Billera and C. Lee, A proof of the sufficiency of McMullen's conditions for $f$-vectors of simplicial convex polytopes, J. Combin. Theory Ser. A 31 (1981), 237-255.

12. A. Björner, Homology of matroids, in Combinatorial Geometries, Vol. II (N. White, ed.), Cambridge University Press, Cambridge, in press.

13. A. Björner and G. Kalai, On $f$-vectors and homology, Proc. NY Acad. Sci, to appear.

14. J. Bokowski and B. Sturmfels, Polytopal and non-polytopal spheres-an algorithmic approach, preprint, TH, Darmstadt, 1985.

15. W. Brown, Enumeration of non-separable planar maps, Canad. J. Math. 15 (1963), 526-545.

16. M. Brückner, Ueber die Ableitung der allgemeinen Polytope and und die nach Isomorphismus verschiedenen Typen der allgemeinen Achtzelle (Oktatope), Verh. Konnik. Konink. Akad. Wetensch. Belgïe, 10 (1909).

17. G. Danaraj and V. Klee, Which spheres are shellable? Ann. Discrete Math. 2 (1978), 33-52.

18. J. Goodman and R. Pollack, There are asymptotically far fewer polytopes than we thought, Bull. Amer. Math. Soc. 14 (1986), 127-129.

19. J. Goodman and R. Pollack, Upper bound for configurations and polytopes in $R^{d}$, Discrete Comput. Geom., to appear.

20. B. Grünbaum, Diagrams and Schlegel diagrams. Abstract 625-112, Notices Amer. Math. Soc. 12 (1965), 578. 
21. B. Grünbaum, Convex Polytopes, Wiley-Interscience, London-New York-Sidney, 1967.

22. B. Grünbaum and V. Sreedharan, An enumeration of simplicial 4 polytopes with 8 vertices, $J$. Combin. Theory 2 (1967), 437-465.

23. G. Kalai, A characterization of $f$-vectors of families of convex sets in $\boldsymbol{R}^{d}$, part II: Sufficiency of Eckhoff's conditions, J. Combin. Theory Ser. A 41 (1986), 167-188.

24. G. Kalai, Algebraic shifting operations and iterated homology groups, to appear.

25. G. Kalai, Rigidity and the lower bound theorem, III: Triangulated manifolds with "few edges", in preparation.

26. V. Klee, The number of vertices of a convex polytope, Canad. J. Math. 16 (1964), 701-720.

27. V. Klee and P. Kleinschmidt, The $d$-step conjecture and its relatives, Math. Oper. Res., to appear.

28. P. Kleinschmidt, Spharen mit wenigen Ecken, Geom. Dedicata 5 (1976), 307-320.

29. P. Kleinschmidt and C. Lee, On $k$-stacked polytopes, Discrete Math. 48 (1984), 125-127.

30. D. J. Kleitman, On Dedekind's problem: the number of monotone Boolean functions, Proc. Amer. Math. Soc. 21 (1969), 677-682.

31. A. D. Korshunov, On the number of monotone Boolean functions, Problemy Kibernet. 38 (1981), 5-108.

32. C. Lee, Two combinatorial properties of a class of simplicial polytopes, Israel J. Math. 47 (1984), 261-269.

33. P. Mani, Spheres with few vertices, J. Combin. Theory Ser. A 13 (1972), 346-352.

34. P. McMullen, The maximum number of faces of a convex polytope, Mathematika 17 (1970), 179-185.

35. P. McMullen, The number of faces of simplicial polytopes, Israel J. Math. 9 (1971), 559-570.

36. P. McMullen, and D. Walkup, A generalized lower bound conjecture for simplicial polytopes, Mathematika 18 (1971), 264-273.

37. U. Pachner, Konstruktionsmethoden und das kombinatorische Homoomorphieproblem fur Triangulationen kompakter semilinearer Mannigfaltigkeiten, preprint.

38. J. Provan and L. Billera, Decompositions of simplicial complexes related to diameters of convex polyhedra, Math. Oper. Res. 5 (1980), 576-595.

39. L. B. Richmond and N. C. Wormald, The asymptotic number of convex polyhedra, Trans. Amer. Math. Soc. 273 (1982), 721-735.

40. I. Shemer, Neighborly polytopes, Israel J. Math. 43 (1982), 291-314.

41. R. Stanley, The upper-bound conjecture and Cohen-Macaulay rings, Stud. Appl. Math. 54 (1975), $135-142$.

42. R. Stanley, Cohen-Macaulay complexes, in Higher Combinatorics (M. Aigner, ed.), 51-62, Reidel, Dordrecht, 1977.

43. R. Stanley, The number of faces of a simplicial convex polytope, Adv. in Math. 35 (1980), 236-238.

44. E. Steinitz and H. Radenmacher Vorlesungen uber die Theorie der Polyeter, Springer-Verlag, Berlin, 1934.

45. W. Tutte, A census of planar triangulations, Canad. J. Math. 14 (1962), 21-38.

46. W. Tutte, On the enumeration of convex polyhedra, J. Combin. Theory Ser. A 28 (1980), 105-126.

Received December 3, 1985, and in revised form October 9, 1986. 